\title{
ON NORMAL APPROXIMATION TO THE DISTRIBUTION OF THE ESTIMATED PROCESS CAPABILITY INDEX $\hat{C}_{p k}$
}

\author{
Yasushi Nagata*
}

\begin{abstract}
Normal approximation to the distribution of the estimated process capability index $\hat{C}_{p k}$ is discussed. The direct normal approximation to $\hat{C}_{p k}$ and an alternative approach are compared in terms of skewness and kurtosis. It is shown that the latter approach improves on the former one.
\end{abstract}

\section{Introduction}

Process capability indices (PCI's) are frequently used in the field of the quality control for the purpose of evaluating whether the processes are satisfactory or not. It has been traditionally thought that PCI's should be used based on large sample size data. It has become more important recently, however to make decisions promptly and consequently PCI's are often calculated from small sample size data. The importance of studying the statistical theory on PCI's is now widely recognized and many studies on PCI's have been done to date. Rodriguez [8] provided a good review and Kotz and Johnson [5] collected almost all the literature which have been done to date on the statistical aspects of PCI's.

Although several types of PCI's have been proposed, in this paper we deal with $C_{p k}$ alone. This is because $C_{p k}$ is a traditional PCI that has been used frequently in Japanese industrial companies.

Assuming that a measured characteristic $X$ is distributed as a normal distribution $N\left(\xi, \sigma^{2}\right)$ and that there are lower and upper specification limits ( $L S L$ and $U S L$, respectively), we define $C_{\mathrm{p} k}$ as follows (see Kotz and Johnson [5] (p. 52)):

$$
\begin{aligned}
C_{p k} & =\frac{\min (U S L-\xi, \xi-L S L)}{3 \sigma} \\
& =\frac{d-|\xi-m|}{3 \sigma},
\end{aligned}
$$

where $d=(U S L-L S L) / 2$ and $m=(L S L+U S L) / 2$. Since $\xi$ and $\sigma$ are unknown parameters, they must be estimated from the data. Let $X_{1}, X_{2}, \ldots, X_{n}$ be independent random variables from the normal distribution $N\left(\xi, \sigma^{2}\right)$. Define $\bar{X}=\sum_{i=1}^{n} X_{i} / n$ and $\hat{\sigma}=\sqrt{\sum_{i=1}^{n}\left(X_{i}-\bar{X}\right)^{2} /(n-1)}$. Then, a natural estimator of $C_{p k}$ is

$$
\hat{C}_{p k}=\frac{d-|\bar{X}-m|}{3 \hat{\sigma}} .
$$

Received December, 1994. Revised April, 1995. Accepted May, 1995

* Faculty of Economics, Okayama University, Tsushima, Okayama 700, Japan 
Chou and Owen [2] derived the distribution function of $\hat{C}_{p k}$, but the formula is very complicated. (Guirguis and Rodriguez [4] provided a computer program for the distribution of $\hat{C}_{p k}$.) Therefore, in order to derive simple formulas for the percentile of $\hat{C}_{p k}$ and for the confidence interval of $C_{p k}$, it is desirable to consider some approximation to the distribution of $\hat{C}_{p k}$. We should note that simple procedures are often favorable for practitioners as long as accuracy is not bad.

Guirguis and Rodriguez [4] presented graphs of the probability density functions of $\hat{C}_{p k}$ and Kotz and Johnson [5] (pp. 66-69) gave several figures of the skewness and the kurtosis of $\hat{C}_{p k}$. These results imply that the distribution of $\hat{C}_{p k}$ is not close to a normal distribution if $n$ is not large, indicating that it is not adequate to consider the direct normal approximation to $\hat{C}_{p k}$ when $n$ is small. (Chan et al. [1] showed that as $n \rightarrow \infty, \sqrt{n}\left(\hat{C}_{p k}-C_{p k}\right)$ is asymptotically distributed as a normal distribution.)

There exists, however, an alternative approximation method. Note that the inequality

$$
\hat{C}_{p k}<c
$$

is equivalent to

$$
\frac{|\bar{X}-m|}{\sigma}+\frac{3 c \hat{\sigma}}{\sigma}>\frac{d}{\sigma} .
$$

The left-hand side of (1.4) can be written as

$$
\frac{1}{\sqrt{n}}|z|+\frac{3 c}{\sqrt{n-1}} \chi_{n-1}
$$

where $z=\sqrt{n}(\bar{X}-m) / \sigma, \chi_{n-1}$ is distributed as a chi distribution with $n-1$ degrees of freedom, and $z$ and $\chi_{n-1}$ are mutually independent. Also note that $z$ is distributed as $N(\sqrt{n} \delta, 1)$, where $\delta=(\xi-m) / \sigma$. Kotz and Johnson [5] (pp. 64-65) described this approach and mentioned that as $n \rightarrow \infty$, the first term of (1.5) becomes negligible compared with the second term of (1.5). This statement is not correct, however unless $\xi=m$ since $|z| / \sqrt{n} \rightarrow|\delta|$ in probability as $n \rightarrow \infty$ and $3 c \chi_{n-1} / \sqrt{n-1}$ tends to $3 c$. On the other hand, $|z|$ is approximately distributed as a normal distribution for large $n$, and we can see from Table 1 that the skewness and the kurtosis of $\chi_{n-1}$ are close to 0 and 3, respectively, the skewness and the kurtosis of a normal distribution. Accordingly, it might be appropriate to consider the normal approximation to (1.5) for large sample size data. In this paper we are interested in the performance of this normal approximation for small sample size data.

This normal approximation can be used to derive percentiles of the distribution of $\hat{C}_{p k}$ (see Section 3). Nagata and Nagahata [7] derived a simple confidence interval formula for $C_{p k}$ based on this approximation. They numerically evaluated the coverage probability of the confidence interval, and showed that this approximation has practical utility. (See also Kushler and Hurley [6] and Zhang et al. $|9|$ for the construction of the confidence interval for $C_{p k}$.)

Nagata and Nagahata |7| did not deal with the direct normal approxima- 
Table 1. Skewness and kurtosis of $\chi_{n-1}$

\begin{tabular}{c|c|c}
\hline$n$ & skewness & kurtosis \\
\hline 10 & .252 & 3.011 \\
20 & .168 & 3.002 \\
30 & .134 & 3.001 \\
40 & .115 & 3.001 \\
50 & .102 & 3.000 \\
100 & .072 & 3.000 \\
\hline
\end{tabular}

tion to $\hat{C}_{p k .}$ Although Kotz and Johnson [5] described the normal approximation to (1.5), they did not compare it with the direct normal approximation. For this reason, we are interested in comparing the normal approximation to (1.5) with the direct normal approximation to $\hat{C}_{p k}$. In Section 2 , we investigate the performance of these approximations in terms of skewness and kurtosis. It is shown that the normal approximation to (1.5) is better than the direct normal approximation to $\hat{C}_{p k}$. In Section 3 , some remarks are given.

\section{Skewness and kurtosis}

Kotz and Johnson [5] (p. 56) derived the formula for the r-th moment of $\hat{C}_{p k}:$

$$
E\left(\hat{C}_{p k}^{r}\right)=\left(\frac{d^{*} \sqrt{f}}{3}\right)^{r} E\left(\chi_{j}^{-r}\right) \sum_{j=0}^{r}(-1)^{j}{ }_{r} C_{j}\left(\frac{1}{d^{*} \sqrt{n}}\right)^{j} E\left(|z|^{j}\right),
$$

where $d^{*}=d / \sigma, f=n-1$, and $z$ and $\chi_{\mathcal{I}}$ were defined in Section 1. As $z$ is distributed as $N(\sqrt{n} \delta, 1)$, the statistic $|z|$ has a folded normal distribution. From Elandt [3], first four moments of $|z|$ are

$$
\begin{aligned}
& E(|z|)=2 \phi(\sqrt{n} \delta)-\sqrt{n} \delta(1-2 \Phi(\sqrt{n} \delta)) \equiv \mu_{1}^{\prime} \\
& E\left(|z|^{2}\right)=1+n \delta^{2} \\
& E\left(|z|^{8}\right)=\left(n \delta^{2}+2\right) \mu_{1}^{\prime}-\sqrt{n} \delta(1-2 \Phi(\sqrt{n} \delta))
\end{aligned}
$$

and

$$
E\left(|z|^{4}\right)=3+6 n \delta^{2}+n^{2} \delta^{4},
$$

where $\phi$ and $\Phi$ are the probability density function and the cumulative distribution function of $N(0,1)$, respectively. Since $E\left(|z|^{r}\right)^{\text {'s }}(r=1 \sim 4)$ are even functions of $\delta$ and all values evaluated in this paper depend on $\delta$ only through $E\left(|z|^{r}\right)^{\prime} \mathrm{s}$, it is sufficient to consider the case $\delta \geq 0$. It is obvious (see, for example, Kotz and Johnson [5] (p. 19)) that

$$
E\left(\chi_{s}^{-r}\right)=\frac{\Gamma((f-r) / 2)}{2^{r / 2} \Gamma(f / 2)},
$$

where $\Gamma(\cdot)$ is the gamma function. From these equations, the skewness and the kurtosis of $\hat{C}_{p k}$ can be calculated, and these depend on $d^{*}, \delta$ and $n$.

Let us denote the skewness and the kurtosis of a random variable $W$ as $S K(W)$ and $K T(W)$, respectively. Kotz and Johnson [5] (pp. 66-69) numeri- 
Table 2. Skewnesses of $\bar{C}_{p k}$ and $Y$ for $n=10$

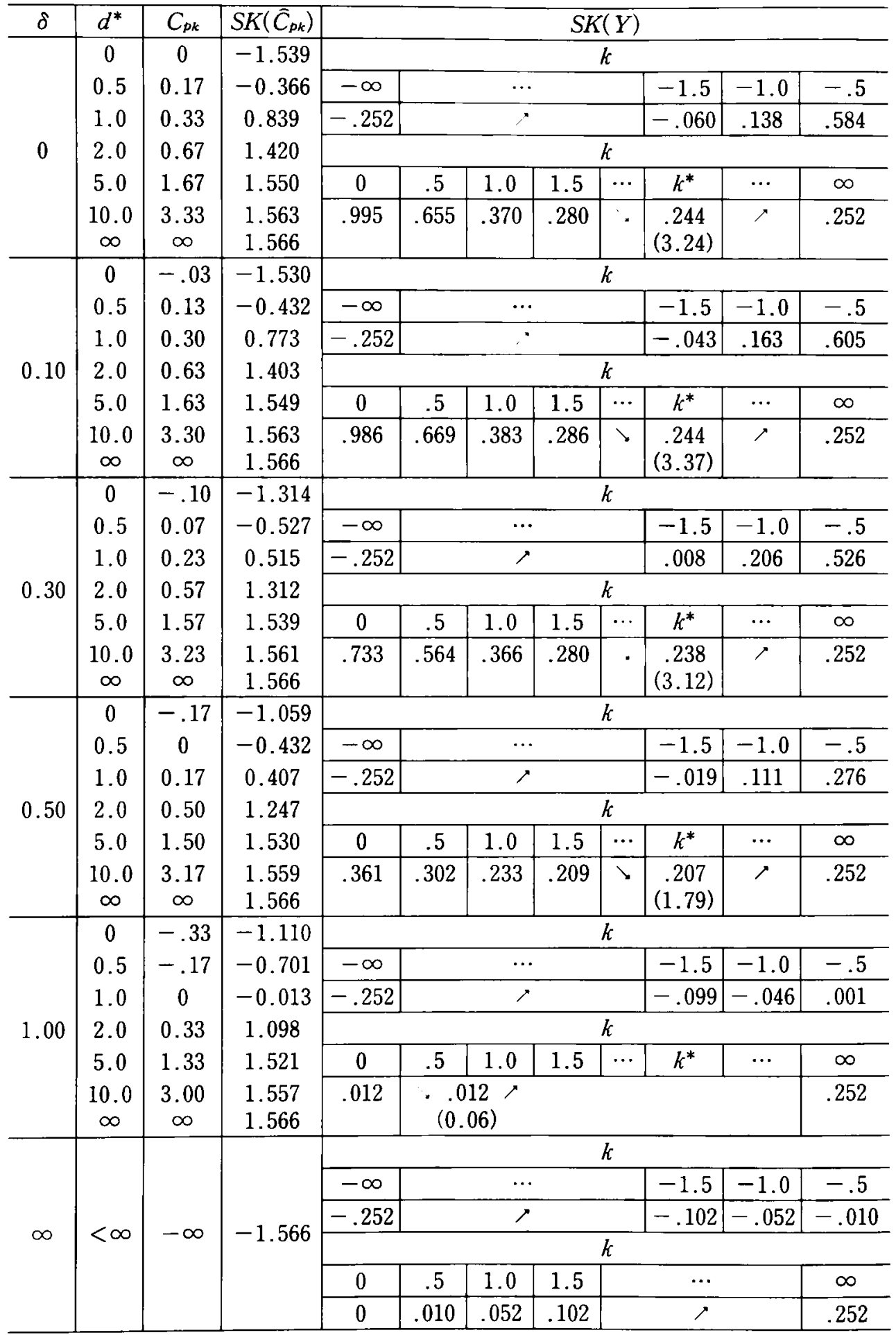

The value of $k^{*}$ is given in the parenthesis. 
Table 3. Skewnesses of $\hat{C}_{p k}$ and $Y$ for $n=30$

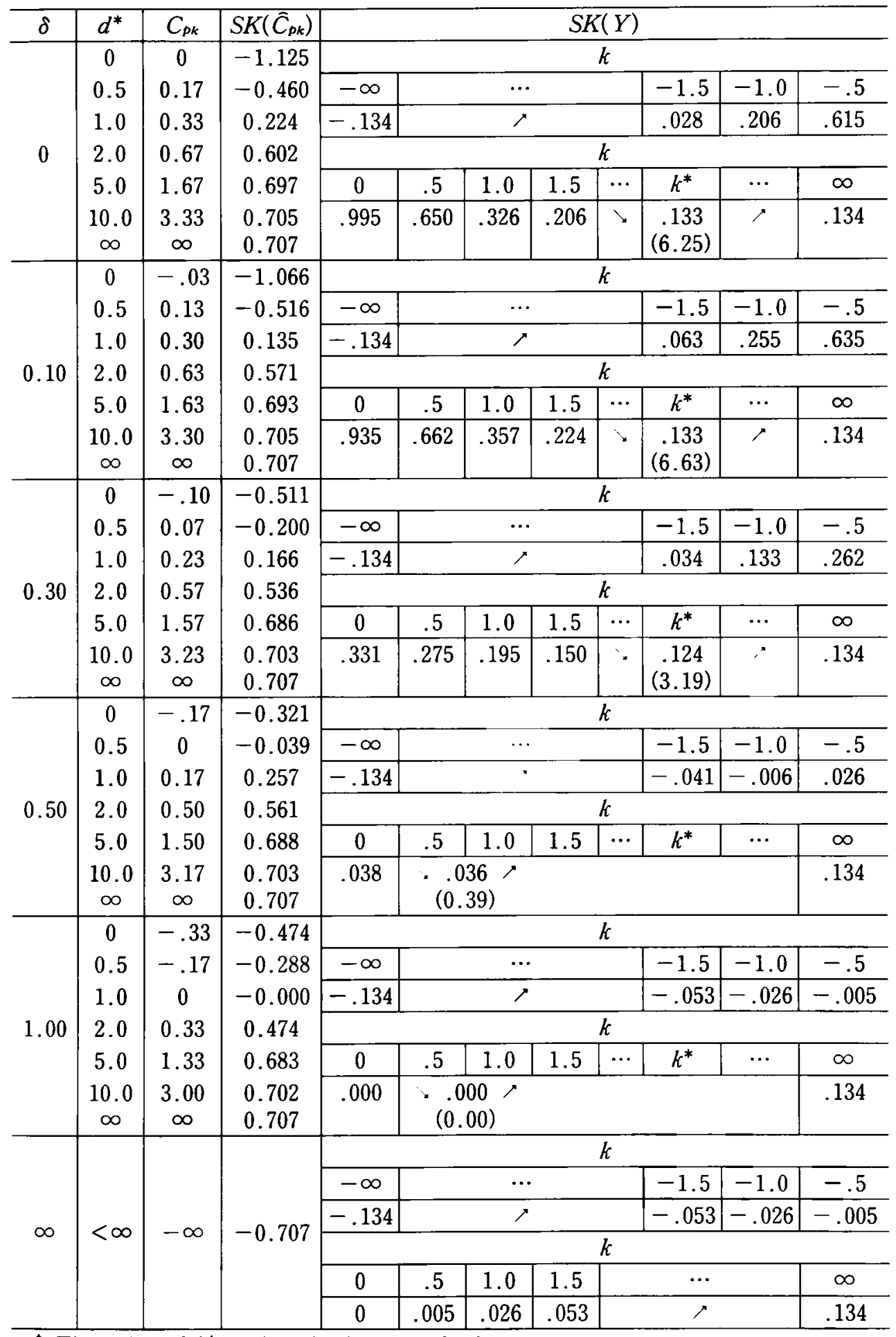

The value of $k^{*}$ is given in the parenthesis. 
Table 4. Skewnesses of $\hat{C}_{p k}$ and $Y$ for $n=100$

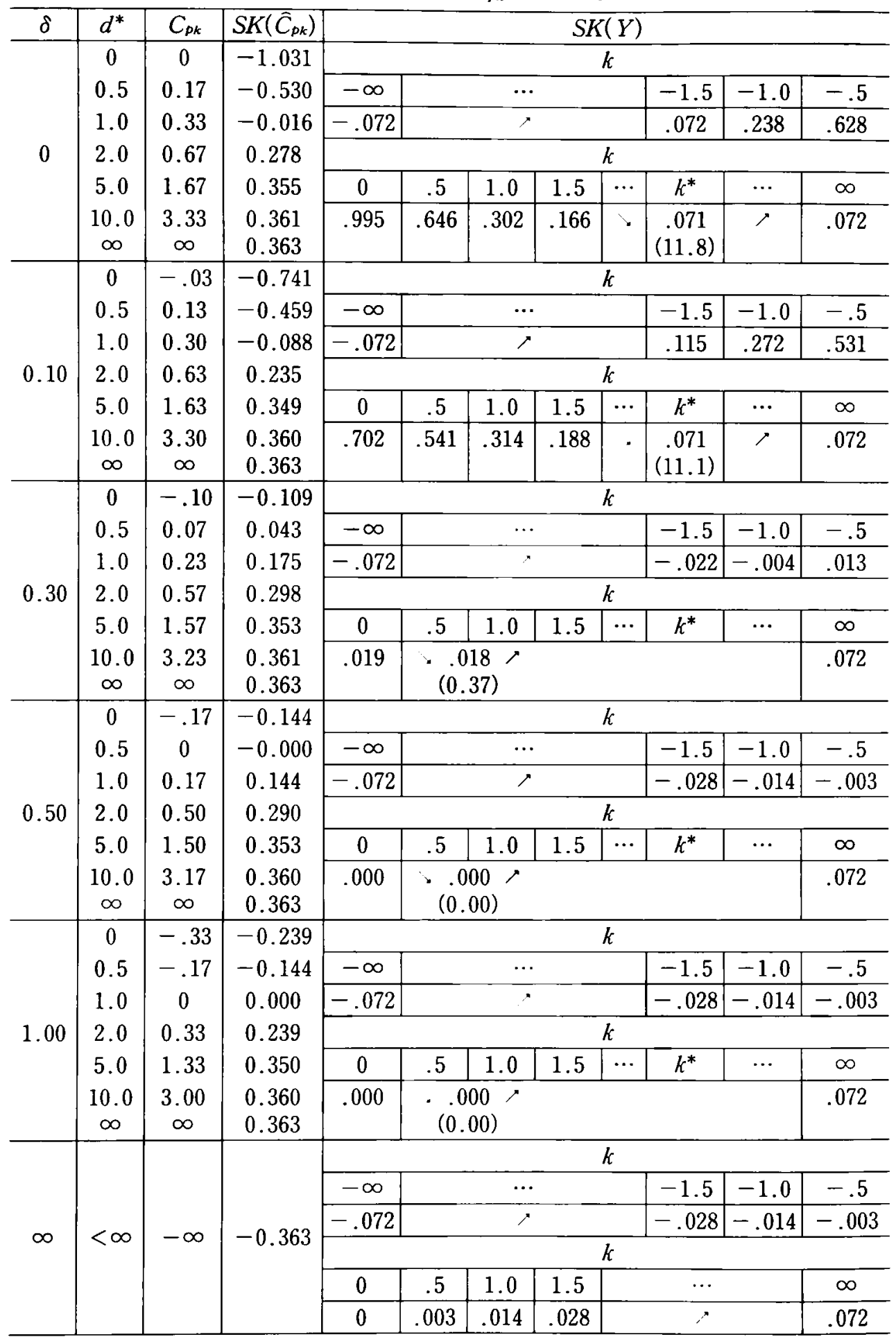

\footnotetext{
- The value of $k^{*}$ is given in the parenthesis.
} 
Table 5. Kurtosises of $\widehat{C}_{p k}$ and $Y$ for $n=10$

\begin{tabular}{|c|c|c|c|c|c|c|c|c|c|c|c|}
\hline \multirow{3}{*}{$\delta$} & \multirow{3}{*}{$d^{*}$} & \multirow{3}{*}{$C_{p k}$} & \multirow{3}{*}{$K T\left(\hat{C}_{p k}\right)$} & \multicolumn{8}{|c|}{$K T(Y)$} \\
\hline & & & & \multicolumn{8}{|c|}{$k$} \\
\hline & & & & 0 & .5 & 1.0 & 1.5 & $\cdots$ & $k^{* *}$ & $\cdots$ & $\infty$ \\
\hline 0 & $\begin{array}{c}0 \\
0.5 \\
1.0 \\
2.0 \\
5.0 \\
10.0 \\
\infty \\
\end{array}$ & $\begin{array}{c}0 \\
0.17 \\
0.33 \\
0.67 \\
1.67 \\
3.33 \\
\infty \\
\end{array}$ & $\begin{array}{l}7.054 \\
4.426 \\
5.804 \\
7.739 \\
8.352 \\
8.419 \\
8.438 \\
\end{array}$ & 3.869 & 3.463 & 3.145 & 3.052 & '. & $\begin{array}{c}3.011 \\
(7.42)\end{array}$ & $r$ & 3.011 \\
\hline 0.10 & $\begin{array}{c}0 \\
0.5 \\
1.0 \\
2.0 \\
5.0 \\
10.0 \\
\infty\end{array}$ & $\begin{array}{c}-.03 \\
0.13 \\
0.30 \\
0.63 \\
1.63 \\
3.30 \\
\infty \\
\end{array}$ & $\begin{array}{l}7.004 \\
4.421 \\
5.632 \\
7.664 \\
8.342 \\
8.417 \\
8.438\end{array}$ & 3.833 & 3.466 & 3.154 & 3.057 & . & $\begin{array}{c}3.011 \\
(7.61)\end{array}$ & $\nearrow$ & 3.011 \\
\hline 0.30 & $\begin{array}{c}0 \\
0.5 \\
1.0 \\
2.0 \\
5.0 \\
10.0 \\
\infty \\
\end{array}$ & $\begin{array}{c}-.10 \\
0.07 \\
0.23 \\
0.57 \\
1.57 \\
3.23 \\
\infty \\
\end{array}$ & $\begin{array}{l}6.023 \\
4.004 \\
4.762 \\
7.232 \\
8.286 \\
8.405 \\
8.438 \\
\end{array}$ & 3.140 & 3.095 & 3.043 & 3.021 & 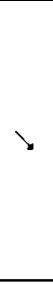 & $\begin{array}{c}3.010 \\
(3.89)\end{array}$ & $\nearrow$ & 3.011 \\
\hline 0.50 & $\begin{array}{c}0 \\
0.5 \\
1.0 \\
2.0 \\
5.0 \\
10.0 \\
\infty\end{array}$ & $\begin{array}{c}-.17 \\
0 \\
0.17 \\
0.50 \\
1.50 \\
3.17 \\
\infty \\
\end{array}$ & $\begin{array}{l}5.382 \\
3.801 \\
4.208 \\
6.843 \\
8.234 \\
8.394 \\
8.438\end{array}$ & 2.697 & 2.775 & 2.889 & 2.953 & & $r$ & & 3.011 \\
\hline 1.00 & $\begin{array}{c}0 \\
0.5 \\
1.0 \\
2.0 \\
5.0 \\
10.0 \\
\infty \\
\end{array}$ & $\begin{array}{c}-.33 \\
-.17 \\
0 \\
0.33 \\
1.33 \\
3.00 \\
\infty \\
\end{array}$ & $\begin{array}{l}6.212 \\
4.948 \\
4.144 \\
6.178 \\
8.174 \\
8.384 \\
8.438 \\
\end{array}$ & 2.960 & 2.969 & 2.948 & 2.995 & & 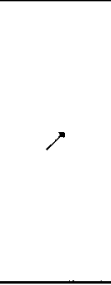 & & 3.011 \\
\hline$\infty$ & $<\infty$ & $-\infty$ & 8.438 & 3 & 3.000 & 3.001 & 3.003 & & $r$ & & 3.011 \\
\hline
\end{tabular}

cally calculated $S K\left(\hat{C}_{p k}\right)$ 's and $K T\left(\hat{C}_{p k}\right)$ 's for $n=30$ and $100, \delta=0,0.075,0.1$, $0.2,0.25$ and 0.4 and $d^{*}=0(0.5) 2.5$, and presented graphs for $\left(S K\left(\hat{C}_{p k}\right)\right.$, $\left.K T\left(\hat{C}_{p k}\right)\right)$. In this paper we also give several values of $S K\left(\hat{C}_{p k}\right)$ and $K T\left(\hat{C}_{p k}\right)$ in order to compare them with the values of the skewness and the kurtosis of (1.5). For $n=10,30$ and $100, \delta=0,0.1,0.3,0.5,1.00$ and $\infty$, and $d^{*}=0,0.5$, $1.0,2.0,5.0,10.0$ and $\infty$, the values of $S K\left(\hat{C}_{p k}\right)$ and $K T\left(\hat{C}_{p k}\right)$ are given in Tables 2-7. 
Table 6. Kurtosises of $\hat{C}_{p k}$ and $Y$ for $n=30$

\begin{tabular}{|c|c|c|c|c|c|c|c|c|c|c|c|}
\hline \multirow{3}{*}{$\delta$} & \multirow{3}{*}{$d^{*}$} & \multirow{3}{*}{$C_{p k}$} & \multirow{3}{*}{$K T\left(\bar{C}_{p k}\right)$} & \multicolumn{8}{|c|}{$K T(Y)$} \\
\hline & & & & \multicolumn{8}{|c|}{$k$} \\
\hline & & & & 0 & .5 & 1.0 & 1.5 & $\cdots$ & $k^{* *}$ & $\cdots$ & $\infty$ \\
\hline 0 & $\begin{array}{c}0 \\
0.5 \\
1.0 \\
2.0 \\
5.0 \\
10.0 \\
\infty \\
\end{array}$ & $\begin{array}{c}0 \\
0.17 \\
0.33 \\
0.67 \\
1.67 \\
3.33 \\
\infty \\
\end{array}$ & $\begin{array}{l}4.451 \\
3.506 \\
3.482 \\
3.820 \\
3.968 \\
3.985 \\
3.989 \\
\end{array}$ & 3.869 & 3.475 & 3.150 & 3.050 & 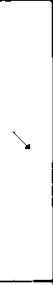 & $\begin{array}{c}3.001 \\
(25.6)\end{array}$ & $\nearrow$ & 3.001 \\
\hline 0.10 & $\begin{array}{c}0 \\
0.5 \\
1.0 \\
2.0 \\
5.0 \\
10.0 \\
\infty \\
\end{array}$ & $\begin{array}{c}-.03 \\
0.13 \\
0.30 \\
0.63 \\
1.63 \\
3.30 \\
\infty\end{array}$ & $\begin{array}{l}4.221 \\
3.431 \\
3.394 \\
3.772 \\
3.961 \\
3.983 \\
3.989 \\
\end{array}$ & 3.656 & 3.403 & 3.148 & 3.054 & $\nu$ & $\begin{array}{c}3.001 \\
(25.2)\end{array}$ & $r$ & 3.001 \\
\hline 0.30 & $\begin{array}{c}0 \\
0.5 \\
1.0 \\
2.0 \\
5.0 \\
10.0 \\
\infty\end{array}$ & $\begin{array}{c}-.10 \\
0.07 \\
0.23 \\
0.57 \\
1.57 \\
3.23 \\
\infty\end{array}$ & $\begin{array}{l}3.126 \\
2.854 \\
3.070 \\
3.637 \\
3.943 \\
3.979 \\
3.989\end{array}$ & 2.688 & 2.764 & 2.878 & 2.943 & & $\nearrow$ & & 3.001 \\
\hline 0.50 & $\begin{array}{c}0 \\
0.5 \\
1.0 \\
2.0 \\
5.0 \\
10.0 \\
\infty\end{array}$ & $\begin{array}{c}-.17 \\
0 \\
0.17 \\
0.50 \\
1.50 \\
3.17 \\
\infty\end{array}$ & $\begin{array}{l}3.290 \\
3.128 \\
3.251 \\
3.678 \\
3.944 \\
3.979 \\
3.989\end{array}$ & 2.896 & 2.918 & 2.955 & 2.978 & & $\nearrow$ & & 3.001 \\
\hline 1.00 & $\begin{array}{c}0 \\
0.5 \\
1.0 \\
2.0 \\
5.0 \\
10.0 \\
\infty \\
\end{array}$ & $\begin{array}{c}-.33 \\
-.17 \\
0 \\
0.33 \\
1.33 \\
3.00 \\
\infty \\
\end{array}$ & $\begin{array}{l}3.561 \\
3.356 \\
3.240 \\
3.561 \\
3.935 \\
3.978 \\
3.989 \\
\end{array}$ & 3.000 & 3.000 & 3.000 & 3.000 & & $\nearrow$ & & 3.001 \\
\hline$\infty$ & $<\infty$ & $-\infty$ & 3.989 & 3 & 3.000 & 3.000 & 3.000 & & $r$ & & 3.001 \\
\hline
\end{tabular}

+ The value of $k^{* *}$ is given in the parenthesis.

Recall that the true parameter value of $C_{p k}$ is equal to $\left(d^{*}-|\delta|\right) / 3$ (see (1.1)). It might be reasonable to confine ourselves to considering the region of realistic values of $C_{p k}$, say $0-3.0$. In fact, when $C_{p k}$ is outside of this region, the inference of $C_{p k}$ is not so important practically.

It can be easily shown that when $\delta$ is fixed, $\lim _{d^{*-\infty}} S K\left(\hat{C}_{p k}\right)=S K\left(\chi_{f}^{-1}\right)$ and $\lim _{d^{* \rightarrow \infty}} K T\left(\hat{C}_{p k}\right)=K T\left(\chi_{\zeta}^{-1}\right)$, and that when $d^{*}$ is fixed, $\lim _{b \rightarrow \infty} S K\left(\hat{C}_{p k}\right)=$ $-S K\left(\chi_{f}^{-1}\right)$ and $\lim _{\delta \rightarrow \infty} K T\left(\hat{C}_{p k}\right)=K T\left(\chi_{f}^{-1}\right)$. 
Table 7. Kurtosises of $\widehat{C}_{p k}$ and $Y$ for $n=100$

\begin{tabular}{|c|c|c|c|c|c|c|c|c|c|c|c|}
\hline \multirow{3}{*}{$\delta$} & \multirow{3}{*}{$d^{*}$} & \multirow{3}{*}{$C_{p k}$} & \multirow{3}{*}{$K T\left(\bar{C}_{p k}\right)$} & \multicolumn{8}{|c|}{$K T(Y)$} \\
\hline & & & & \multicolumn{8}{|c|}{$k$} \\
\hline & & & & 0 & .5 & 1.0 & 1.5 & $\cdots$ & $k^{* *}$ & $\ldots$ & $\infty$ \\
\hline 0 & $\begin{array}{c}0 \\
0.5 \\
1.0 \\
2.0 \\
5.0 \\
10.0 \\
\infty\end{array}$ & $\begin{array}{c}0 \\
0.17 \\
0.33 \\
0.67 \\
1.67 \\
3.33 \\
\infty\end{array}$ & $\begin{array}{l}4.019 \\
3.398 \\
3.168 \\
3.204 \\
3.248 \\
3.254 \\
3.255\end{array}$ & 3.869 & 3.479 & 3.153 & 3.051 & . & $\begin{array}{c}3.000 \\
(89.8)\end{array}$ & $\therefore$ & 3.000 \\
\hline 0.10 & $\begin{array}{c}0 \\
0.5 \\
1.0 \\
2.0 \\
5.0 \\
10.0 \\
\infty\end{array}$ & $\begin{array}{c}-.03 \\
0.13 \\
0.30 \\
0.63 \\
1.63 \\
3.30 \\
\infty \\
\end{array}$ & $\begin{array}{l}3.208 \\
2.983 \\
2.997 \\
3.152 \\
3.242 \\
3.253 \\
3.255 \\
\end{array}$ & 3.079 & 3.055 & 3.025 & 3.010 & . & $\begin{array}{c}3.000 \\
(35.9)\end{array}$ & $\nearrow$ & 3.000 \\
\hline 0.30 & $\begin{array}{c}0 \\
0.5 \\
1.0 \\
2.0 \\
5.0 \\
10.0 \\
\infty \\
\end{array}$ & $\begin{array}{c}-.10 \\
0.07 \\
0.23 \\
0.57 \\
1.57 \\
3.23 \\
\infty\end{array}$ & $\begin{array}{l}3.022 \\
3.008 \\
3.068 \\
3.180 \\
3.243 \\
3.252 \\
3.255 \\
\end{array}$ & 2.941 & 2.953 & 2.974 & 2.987 & & $r$ & & 3.000 \\
\hline 0.50 & $\begin{array}{c}0 \\
0.5 \\
1.0 \\
2.0 \\
5.0 \\
10.0 \\
\infty \\
\end{array}$ & $\begin{array}{c}-.17 \\
0 \\
0.17 \\
0.50 \\
1.50 \\
3.17 \\
\infty \\
\end{array}$ & $\begin{array}{l}3.091 \\
3.063 \\
3.091 \\
3.182 \\
3.243 \\
3.252 \\
3.255 \\
\end{array}$ & 3.000 & 3.000 & 3.000 & 3.000 & & $\nearrow$ & & 3.000 \\
\hline 1.00 & $\begin{array}{c}0 \\
0.5 \\
1.0 \\
2.0 \\
5.0 \\
10.0 \\
\infty\end{array}$ & $\begin{array}{c}-.33 \\
-.17 \\
0 \\
0.33 \\
1.33 \\
3.00 \\
\infty\end{array}$ & $\begin{array}{l}3.142 \\
3.091 \\
3.063 \\
3.142 \\
3.240 \\
3.252 \\
3.255\end{array}$ & 3.000 & 3.000 & 3.000 & 3.000 & & - & & 3.000 \\
\hline$\infty$ & $<\infty$ & $-\infty$ & 3.255 & 3 & 3.000 & 3.000 & 3.000 & & $r$ & & 3.000 \\
\hline
\end{tabular}

We can check the values in our tables by comparing them for $n=30$ and 100 with the values in the figures given by Kotz and Johnson [5].

From the figures in Kotz and Johnson [5] or our tables, we observe that $S K\left(\hat{C}_{p k x}\right)$ and $K T\left(\hat{C}_{p k}\right)$ vary in wide range as $d^{*}$ and $\delta$ increase, but that for larger $n\left(S K\left(\hat{C}_{p k}\right), K T\left(\hat{C}_{p k}\right)\right)$ is getting closer to $(0,3)$.

Now, let us derive the skewness and the kurtosis of (1.5), which is the main purpose of this paper. For simplicity of notation, we rewrite (1.5) as 


$$
Y=\frac{1}{\sqrt{n}}|z|+\frac{k}{\sqrt{f}} X_{r},
$$

where $k=3 c$. Denoting the $r$-th central moments of $|z|, \chi_{r}$ and $Y$ as $\mu_{r}, \nu_{r}$ and $\rho_{r}$, respectively, we can obtain

$$
\begin{aligned}
& \rho_{2}=\frac{1}{n} \mu_{2}+\frac{k^{2}}{f} \nu_{2} \\
& \rho_{3}=\frac{1}{n^{3 / 2}} \mu_{3}+\frac{k^{8}}{f^{3 / 2}} \nu_{3}
\end{aligned}
$$

and

$$
\rho_{4}=\frac{1}{n^{2}} \mu_{4}+\frac{6 k^{2}}{n f} \mu_{2} \nu_{2}+\frac{k^{4}}{f^{2}} \nu_{4}
$$

Since we can calculate $\mu_{r}(r=2-4)$ from (2.2)-(2.5) and $\nu_{r}$ from (2.6), we can numerically evaluate $\rho_{r}(r=2-4), S K(Y) \quad\left(=\rho_{s} / \rho_{2}^{3 / 2}\right)$ and $K T(Y) \quad\left(=\rho_{4} / \rho_{2}^{2}\right)$. Note that $\rho_{r}$ depends on $\delta, k$ and $n$, and that it does not depend on $d^{*}$.

First, let us consider the skewness of $Y$. It follows that $\lim _{k \rightarrow-\infty} S K(Y)$ $=-S K\left(\chi_{\jmath}\right), \lim _{k \rightarrow 0} S K(Y)=S K(|z|)$ and $\lim _{k \rightarrow \infty} S K(Y)=S K\left(\chi_{\jmath}\right)$. Furthermore, we have

$$
\frac{\partial S K(Y)}{\partial k}=\frac{3 k}{n f \rho_{2}^{6 / 2}}\left(\frac{\mu_{2} \nu_{8}}{\sqrt{f}} k-\frac{\mu_{8} \nu_{2}}{\sqrt{n}}\right) .
$$

Denote $k^{*}=\sqrt{f} \mu_{s} \nu_{2} /\left(\sqrt{n} \mu_{2} \nu_{3}\right)$. Note that $\mu_{8}>0$ and $\nu_{8}>0$ for $\delta<\infty$ can be confirmed from the columns at $k=0$ and $\infty$ in Tables 2-4, respectively, so that $k^{*}>0$ for $\delta<\infty$. Therefore, $S K(Y)$ attains a local maximum at $k=0$ and a local minimum at $k=k^{*}, S K(Y)$ increases with $k \in(-\infty, 0) \cup\left(k^{*}, \infty\right)$, and decreases with $k \in\left(0, k^{*}\right)$.

We can also show that

$$
\lim _{d \rightarrow \infty} S K(Y)=\frac{\frac{k^{8}}{f^{3 / 2} \nu_{3}}}{\left(\frac{1}{n}+\frac{k^{2}}{f} \nu_{2}\right)^{3 / 2}} .
$$

It follows that the right-hand side of (2.12) increases as $k$ increases and attains its infimum value $-S K\left(\chi_{f}\right)$ at $k=-\infty$ and its supremum value $S K\left(\chi_{f}\right)$ at $k=\infty$.

In Tables 2-4, the values of $S K(Y)$ are given for the selected values of $\delta$, $k$ and $n$. From these tables we can observe the following points: (a) $S K(|z|)$ $(=S K(Y)$ at $k=0)$ tends to 0 as $\delta$ increases, which was also observed by Elandt [3] (see his Table 1). (b) Every maximum value of $|S K(Y)|$ with respect to $k$ for each fixed value of $\delta$ is smaller than that of $\left|S K\left(\hat{C}_{p k x}\right)\right|$ with respect to $d^{*}$. (c) $|S K(Y)|$ becomes much smaller than $\left|S K\left(\hat{C}_{p k}\right)\right|$ as $\delta$ and/or $|k|$ increase. (d) $S K(Y)$ is getting closer to 0 for larger $n$.

Next, let us consider the kurtosis of $Y$. First of all, note that $K T(Y)$ is an even function of $k$ (see (2.8) and (2.10)). It follows that $\lim _{k \rightarrow 0} K T(Y)=$ $K T(|z|)$ and $\lim _{k \rightarrow \infty} K T(Y)=K T\left(\chi_{f}\right)$. Furthermore, we have 


$$
\frac{\partial K T(Y)}{\partial k}=\frac{4 \mu_{2} \nu_{2}^{2}}{n f^{2} \rho_{2}^{3}}\left(K T\left(\chi_{\jmath}\right)-3\right) k\left\{k^{2}-\frac{f \mu_{2}(K T(|z|)-3)}{n \nu_{2}\left(K T\left(\chi_{f}\right)-3\right)}\right\} .
$$

Since $K T\left(\chi_{f}\right)>3$ (see Table 1), it follows that if $K T(|z|) \leq 3, K T(Y)$ increases as $k$ increases, and that if $K T(|z|)>3, K T(Y)$ decreases with $k \in\left(0, k^{* *}\right)$, attains the local minimum at $k=k^{* *}$ and increases with $k \in\left(k^{* *}, \infty\right)$, where

$$
k^{* *}=\sqrt{\frac{f \mu_{2}(K T(|z|)-3)}{n \nu_{2}\left(K T\left(\chi_{\jmath}\right)-3\right)}} .
$$

We can also show that

$$
\lim _{i \rightarrow \infty} K T(Y)=\frac{\frac{3}{n^{2}}+\frac{6 k^{2}}{n f} \nu_{2}+\frac{k^{4}}{f^{2}} \nu_{4}}{\left\{\frac{1}{n}+\frac{k^{2}}{f} \nu_{2}\right\}^{2}}
$$

It follows that the right-hand side of $(2.15)$ increases as $k$ increases.

In Tables 5-7, the values of $K T(Y)$ are given for the selected values of $\delta$, $k$ and $n$. We can see from these tables the following points: (a) $K T(|z|)$ $(=K T(Y)$ at $k=0)$ initially decreases as $\delta$ increases from zero, attaining its minimum (which is below 3 ), and then tends to 3 with $\delta$ (observed by Elandt [3], see his Table 1). (b) $K T(Y)$ becomes closer to 3 than $K T\left(\hat{C}_{p k}\right)$ as $\delta$ and/or $|k|$ increase. (c) $K T(Y)$ is much closer to 3 than $K T\left(\hat{C}_{p k}\right)$ for smaller $n$.

\section{Concluding remarks}

This paper investigated the skewnesses and the kurtosises of $\hat{C}_{p k}$ and $Y$. From the results in the previous section, we can conclude that if $\delta$ and/or $|k|$ are large, the distribution of $Y$ is much closer to a normal distribution than that of $\hat{C}_{p k}$. As $\delta$ is unknown, what size of $k$ is enough for a normal approximation, and whether or not this size can be realized in practical applications are our interests. In this section we consider these problems briefly.

Roughly speaking, we might claim from our results that when $|k| \geq 1.5$, the normal approximation to $Y$ is applicable. (It can be observed from Tables 2-7 that if $|k| \geq 1.5, \max _{\delta}|S K(Y)| \leq 0.30$ and $\max _{\delta}|K T(Y)-3| \leq 0.06$.)

Define $q_{\alpha}$ as the $100 \alpha$ percentile of the distribution of $\hat{C}_{p k}$. That is, $q_{\alpha}$ satisfies

$$
\operatorname{Pr}\left(\hat{C}_{p k} \leq q_{\alpha}\right)=\alpha .
$$

Nagata and Nagahata [7] used the normal approximation to (1.5) and derived a simple approximate formula for $q_{\alpha}$ :

$$
q_{\alpha} \approx \frac{C_{p k}+z_{\alpha} \sqrt{\frac{C_{p k}^{2}}{2 f}+\left(1-\frac{z_{\alpha}^{2}}{2 f}\right) /(9 n)}}{1-z_{a}^{2} /(2 f)},
$$

where $z_{\alpha}$ is the $100 \alpha$ percentile of $N(0,1)$. Recalling $k=3 q_{\alpha}$ (see (1.3), (1.5) and (2.7)), we can consider the approximate size of $k$ which is realized in practical applications. These approximate values of $k$ based on (3.2) are given in Table 8. From this table, it is observed that only when $C_{p k}=0.5$ and $\alpha<0.50$, the 
Table 8. Approximate values of $k$ for percentiles

\begin{tabular}{c|c|c|c|c|c|c|c|c}
\hline \multirow{3}{*}{$n$} & \multirow{2}{*}{$C_{p k}$} & \multicolumn{7}{|c}{$\alpha$} \\
\cline { 3 - 8 } & & .05 & .10 & .25 & .50 & .75 & .90 & .95 \\
\hline \multirow{4}{*}{10} & .5 & .88 & 1.00 & 1.21 & 1.50 & 1.86 & 2.31 & 2.65 \\
& 1.0 & 2.05 & 2.22 & 2.54 & 3.00 & 3.61 & 4.39 & 5.01 \\
& 1.5 & 3.17 & 3.40 & 3.85 & 4.50 & 5.38 & 6.51 & 7.43 \\
& 2.0 & 4.27 & 4.56 & 5.15 & 6.00 & 7.16 & 8.64 & 9.86 \\
& 2.5 & 5.36 & 5.72 & 6.45 & 7.50 & 8.94 & 10.78 & 12.30 \\
\hline \multirow{5}{*}{30} & .5 & 1.12 & 1.19 & 1.33 & 1.50 & 1.69 & 1.90 & 2.03 \\
& 1.0 & 2.40 & 2.52 & 2.73 & 3.00 & 3.32 & 3.66 & 3.89 \\
& 1.5 & 3.66 & 3.82 & 4.12 & 4.50 & 4.96 & 5.45 & 5.79 \\
& 2.0 & 4.90 & 5.11 & 5.50 & 6.00 & 6.60 & 7.24 & 7.69 \\
& 2.5 & 6.14 & 6.40 & 6.88 & 7.50 & 8.24 & 9.04 & 9.59 \\
\hline \multirow{1}{*}{100} & .5 & 1.28 & 1.33 & 1.40 & 1.50 & 1.60 & 1.70 & 1.76 \\
& 1.0 & 2.65 & 2.72 & 2.85 & 3.00 & 3.17 & 3.33 & 3.43 \\
& 1.5 & 4.00 & 4.10 & 4.28 & 4.50 & 4.74 & 4.97 & 5.12 \\
& 2.0 & 5.35 & 5.48 & 5.72 & 6.00 & 6.31 & 6.62 & 6.81 \\
& 2.5 & 6.70 & 6.86 & 7.15 & 7.50 & 7.88 & 8.26 & 8.51 \\
\hline
\end{tabular}

rule of thumb (i.e. $|k| \geq 1.5$ ) is not satisfied.

In conclusion, the normal approximation to (1.5) might be useful in deriving simple approximation formulas. (Of course, some further devices which include the bias correction etc. would lead to more accurate approximation. Nagata and Nagahata [7] have previously considered the bias correction and derived the conservative confidence interval for $C_{p k \text {. }}$ )

\section{Acknowledgement}

The author would like to thank the referees for their valuable comments.

\section{REFERENCES}

[1] Chan, L. K., Xiong, Z. and Zhang, D. (1990). On the asymptotic distributions of some process capability indices, Communications in Statistics-Theory and Methods, 19, 11-18.

[2] Chou, Y. M. and Owen, D. B. (1989). On the distribution of the estimated process capability indices, Communications in Statistics-Theory and Methods, 18, 4549-4560.

[ 3 ] Elandt, R. C. (1961). The folded normal distribution: two methods of estimating parameters from moments, Technometrics, 3, 551-562.

[4] Guirguis, G. H. and Rodriguez, R. N. (1992). Computation of Owen's $Q$ function applied to process capability analysis, Journal of Quality Technology, 24, 236-246.

[5] Kotz, S. and Johnson, N. L. (1993). Process Capability Indices, Chapman and Hall.

[6] Kushler, R. H. and Hurley, P. (1992). Confidence bounds for capability indices, Journal of Quality Technology, 24, 188-195. 
[ 7 ] Nagata, Y. and Nagahata, H. (1992). Approximation formulas for the confidence intervals of process capability indices, Reports of Statistical Application Research, JUSE, 39(3), 15-29.

[8] Rodriguez, R. N. (1992). Recent developments in process capability analysis, Journal of Quality Technology, 24, 176-187.

[9] Zhang, N. F., Stenback, G. A. and Wardrop, D. M. (1990). Interval estimation of process capability index $\hat{C}_{p k}$, Communications in Statistics-Theory and Methods, 19, 4455-4470. 